\title{
Lack of Pharmacokinetic Interactions Between Dapagliflozin and Simvastatin, Valsartan, Warfarin, or Digoxin
}

Sreeneeranj Kasichayanula · Ming Chang · Xiaoni Liu $\cdot$ Wen-Chyi Shyu $\cdot$ Steven C. Griffen · Frank P. LaCreta ·

David W. Boulton

To view enhanced content go to www.advancesintherapy.com

Received: December 1, 2011 / Published online: January 19, 2012

(c) The Author(s) 2012. This article is published with open access at Springerlink.com

\section{ABSTRACT}

Introduction: Coronary heart disease, stroke, and peripheral vascular disease are the most common causes of mortality in patients with type 2 diabetes mellitus (T2DM). The aim of these studies was to assess the potential

S. Kasichayanula ( $\square)$

Discovery Medicine and Clinical Pharmacology, Bristol-Myers Squibb Company, PO Box 4000, Princeton, NJ 08543-4000, USA.

Email: sreeneeranj.kasichayanula@bms.com

M. Chang · S. C. Griffen · F. P. LaCreta · D. W. Boulton Discovery Medicine and Clinical Pharmacology, Princeton, NJ, 08643-4000, USA

X. Liu

Global Biometric Sciences, Bristol-Myers Squibb Company, Princeton, NJ, USA

W-C. Shyu

Discovery Medicine and Clinical Pharmacology, Princeton, NJ, 08643-4000, USA

Present Address: Millennium Pharmaceuticals, Cambridge, MA, USA

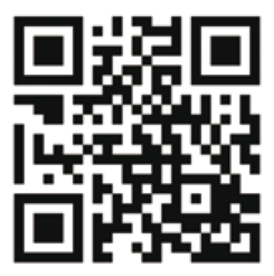

Enhanced content for Advances in Therapy articles is available on the journal web site: www.advancesintherapy.com for pharmacokinetic interaction between dapagliflozin, a sodium glucose co-transporter-2 inhibitor being developed for the treatment of T2DM, and four medications commonly prescribed in patients with T2DM and cardiovascular disease: simvastatin, valsartan, warfarin, and digoxin.

Methods: Potential pharmacokinetic interactions between $20 \mathrm{mg}$ dapagliflozin, $40 \mathrm{mg}$ simvastatin, or $320 \mathrm{mg}$ valsartan were assessed in an openlabel, randomized, five-period, five-treatment, unbalanced crossover study in 24 healthy subjects. In a second study, the effects of steadystate dapagliflozin on the pharmacokinetics of $25 \mathrm{mg}$ warfarin or $0.25 \mathrm{mg}$ digoxin were assessed in an open-label, randomized, two-period, two-treatment crossover study in 30 healthy subjects divided into two cohorts. The potential pharmacodynamic interaction between dapagliflozin and warfarin was also evaluated.

Results: All treatments were well tolerated. Neither simvastatin nor valsartan had any clinically meaningful effect on the pharmacokinetics of dapagliflozin. Dapagliflozin increased the area under the curve for simvastatin, simvastatin acid, and valsartan by approximately $19 \%, 30 \%$, and $6 \%$, respectively, and decreased the maximum observed plasma 
concentration of valsartan by approximately $6 \%$. These effects were not considered clinically meaningful. In addition, dapagliflozin had no effect on the pharmacokinetics of either digoxin or warfarin. The pharmacodynamics of warfarin were also unaffected by dapagliflozin.

Conclusion: In these studies the co-administration of dapagliflozin and simvastatin, valsartan, warfarin, or digoxin was well tolerated without clinically meaningful drug-drug interaction.

Keywords: dapagliflozin; digoxin; pharmacokinetics; pharmacodynamics; simvastatin; sodium glucose co-transporter-2; type 2 diabetes; valsartan; warfarin

\section{INTRODUCTION}

The global burden of diabetes is daunting: there were an estimated 285 million sufferers worldwide in 2010 [1,2], and this number is predicted to rise to 438 million by 2030 . Type 2 diabetes mellitus (T2DM) is the predominant form, accounting for approximately $90 \%$ of all cases of diabetes [3]. T2DM occurs primarily in economically developing and newly industrialized countries, where it has reached epidemic proportions, largely as a result of urbanization, associated lifestyle changes, and consequent obesity [4]. Increasing body mass index (BMI) is associated with an increased prevalence of T2DM, dyslipidemia, and hypertension [5]. The simultaneous achievement of glycemic, blood pressure, and low-density lipoprotein targets is pivotal to the management of T2DM to avoid cardiovascular disease and premature death [6].

The medical management of hyperglycemia in T2DM concentrates not only on the use of lifestyle-directed interventions, but also on pharmacologic interventions, such as insulin, sulfonylureas, and metformin [7]. Recently, the search for insulin-independent mechanisms for glycemic control in T2DM has resulted in the development of a novel approach - inducing renal glucose excretion pharmacologically by inhibiting the reabsorption of glucose from the glomerular filtrate. Under conditions of normoglycemia, virtually all glucose in the glomerular filtrate is reabsorbed in the proximal tubules, mostly via sodium glucose co-transporter-2 (SGLT2) [8]. Dapagliflozin is a first-in-class oral, selective, reversible inhibitor of SGLT2 in development for the treatment of T2DM [9]. Studies in healthy and diabetic rats have demonstrated that dapagliflozin improves glucose homeostasis [10], and dosedependent urinary glucose excretion is induced by dapagliflozin in humans $[11,12]$. Clinical studies have also demonstrated the efficacy of dapagliflozin in the treatment of T2DM [12-17].

There is a clear epidemiologic and pathophysiologic link between both microand macro-vascular disease and T2DM [18]. Coronary heart disease, stroke, and peripheral vascular disease are the most common causes of mortality in patients with T2DM [19]. Cardiovascular disease, such as atherosclerosis or congestive heart failure, is often present in patients with T2DM [20,21]. For this reason, in addition to the rigorous management of hyperglycemia, dyslipidemia, and hypertension, T2DM patients with cardiovascular disease may require treatment with commonly prescribed cardiovascular drugs, such as warfarin and digoxin, to prevent future major cardiovascular events and improve prognosis.

Because of the likelihood of any new T2DM therapy being co-administered with cardiovascular drugs, the two studies reported here were conducted to investigate the safety profile of co-administration and the potential for pharmacokinetic or pharmacodynamic 
interaction between dapagliflozin and the commonly prescribed dyslipidemic drug simvastatin or the angiotensin II receptor blocker valsartan, used for the treatment of hypertension (study 1; clinicaltrials. gov identifier: NCT00839683) and between dapagliflozin and the anticoagulant warfarin or the cardiac glycoside digoxin (study 2; clinicaltrials.gov identifier: NCT00904176).

In-vitro pharmacologic studies suggest that dapagliflozin is primarily metabolized via glucuronidation by uridine diphosphate glucuronyl transferase (UGT1A9 enzyme). Under in-vitro conditions, dapagliflozin was not found to be an inhibitor or an inducer of human cytochrome (CYP) isozymes and, although it proved to be a substrate for P-glycoprotein (P-gp), it has not been demonstrated to be a significant P-gp inhibitor. Thus, dapagliflozin was not anticipated to significantly affect the pharmacokinetics of co-administered drugs that are CYP or P-gp substrates [22].

Valsartan is minimally metabolized in humans, with no involvement of CYP isozymes [23]. In addition, there is no evidence to indicate that its rapid absorption is affected by P-gp. Simvastatin is metabolized predominantly via CYP3A4 [24] and, while a variety of simvastatin drug-drug interactions have been observed, significant interactions that are clinically evident remain rare [25]. S-warfarin, the enantiomer that is primarily responsible for warfarin's anticoagulant effects, is principally metabolized by CYP2C9 [26]. The metabolism of digoxin is reported to be minimal and is also not dependent on CYP isozymes. Digoxin is excreted in urine and bile largely unchanged [27]. The gastrointestinal absorption of digoxin, however, does appear to be mediated by P-gp [28].

Small alterations in the absorption, free fraction, or clearance of these cardiovascular drugs - and in particular warfarin and digoxin, both of which have a narrow therapeutic index - could lead to a decrease in therapeutic effect, or to potential toxicity. Therefore, although pharmacokinetic interactions are not predicted on the basis of the routes of metabolism, it is important to confirm a lack of clinically significant interaction via clinical pharmacokinetic studies. In addition, as there is evidence for pharmacodynamic interaction of some drugs (for example, rosuvastatin [29]) with warfarin without concomitant changes in pharmacokinetics, pharmacodynamic parameters were also evaluated for warfarin (plasma prothrombin time) in the presence and absence of dapagliflozin.

\section{MATERIALS AND METHODS}

\section{Subjects}

For both studies, male and female subjects deemed to be healthy on the basis of medical history, physical examination, 12-lead electrocardiogram, and clinical laboratory evaluations were eligible. Other inclusion criteria were age in the range of $18-45$ years and a BMI of $18-32 \mathrm{~kg} / \mathrm{m}^{2}$. No woman of child-bearing potential who was breast-feeding, pregnant, or not using an acceptable method of contraception was eligible. Exclusion criteria included a urinary tract infection in the 3 months preceding screening, a history of recurrent or recent vulvovaginal mycotic infections, and current smoking. For study 2 , subjects with an international normalized ratio (INR) value above the upper limit of normal on day 11 of period 1 were excluded from entering period 2 of the study.

\section{Study Designs}

Protocol, protocol amendments, and informed consent were approved by the respective 
institutional review boards. Both studies were carried out in accordance with the Guidelines for Good Clinical Practice and adhered to the principles of the Declaration of Helsinki and the US Food and Drug Administration (FDA) Code of Federal Regulations. Written informed consent was obtained from all participants prior to enrollment. Healthy subjects were screened within 21 days (study 1 ) and 28 days (study 2) prior to initial dosing to establish eligibility. Eligible subjects were admitted to the clinical research facility on day -1 of period 1 and remained there until discharge on day 20 (study 1) or day 23 (study 2).

Study 1 explored whether dapagliflozin had any effects on the pharmacokinetics of simvastatin or valsartan, and also whether simvastatin or valsartan affected the pharmacokinetics of dapagliflozin. Study 2 evaluated the effects of dapagliflozin on the pharmacokinetics of warfarin or digoxin, and also assessed the effects of dapagliflozin on the pharmacodynamics of warfarin. No evaluation of dapagliflozin alone was made in study 2 , and there was no assessment of the effect of warfarin or digoxin on the pharmacokinetics of dapagliflozin in this study.

\section{Study 1}

This was an open-label, randomized, fiveperiod, five-treatment, unbalanced crossover study (Fig. 1a). Twenty-four enrolled subjects (23 men and one woman) were randomized to one of 12 treatment sequences and received the following five single-dose oral treatments: $20 \mathrm{mg}$ dapagliflozin alone, $40 \mathrm{mg}$ simvastatin alone, $20 \mathrm{mg}$ dapagliflozin plus $40 \mathrm{mg}$ simvastatin, $320 \mathrm{mg}$ valsartan alone, and $20 \mathrm{mg}$ dapagliflozin plus $320 \mathrm{mg}$ valsartan. Subjects were required to fast for 10 hours before through to 4 hours after study drug administration. All study medication was administered at time 0 hours with water $(240 \mathrm{~mL})$. Serial blood samples (a total of three 4-mL samples for each time point) were collected for up to 72 hours after dosing for the pharmacokinetic assessment of dapagliflozin; simvastatin; its major active metabolite, simvastatin acid; and valsartan. Samples destined for dapagliflozin and valsartan analysis were collected in di-potassium ethylenediaminetetraacetic acid ( $\left.\mathrm{K}_{2} \mathrm{EDTA}\right)$ tubes, and those for simvastatin and simvastatin acid analysis were collected in lithium-heparin tubes. Plasma was isolated by centrifugation and stored at $-20^{\circ} \mathrm{C}$ for dapagliflozin and $-70^{\circ} \mathrm{C}$ for valsartan, simvastatin, and simvastatin acid until analyzed.

\section{Study 2}

An open-label, randomized, two-period, two-treatment crossover study (Fig. 1b) was employed to investigate the effect of steadystate administration of dapagliflozin on the pharmacokinetics of the R- and S-enantiomers of warfarin (S-warfarin has approximately five times the potency of the R-isomer with respect to vitamin $\mathrm{K}$ antagonism) [30] or digoxin. A total of 30 subjects (all men) were randomized to one of two study treatments: warfarin (cohort 1, comprising 14 subjects) and digoxin (cohort 2 , comprising 16 subjects). Subjects in cohort 1 were randomized to receive an oral loading dose of $20 \mathrm{mg}$ dapagliflozin on day 1 followed by oral administration of $10 \mathrm{mg}$ dapagliflozin once daily for 7 days (days 2-8), and co-administration with a single oral dose of $25 \mathrm{mg}$ warfarin on day 2 , or a single oral dose of $25 \mathrm{mg}$ warfarin on day 2. The loading dose of dapagliflozin was selected to ensure that sufficient exposure was achieved at the time of dosing with warfarin or digoxin (day 2). Since both warfarin and digoxin have long elimination half-lives $\left(t_{1 / 2}\right)$ relative to dapagliflozin, repeat daily dosing of dapagliflozin on days 2-8 was necessary after co-administration 
a)

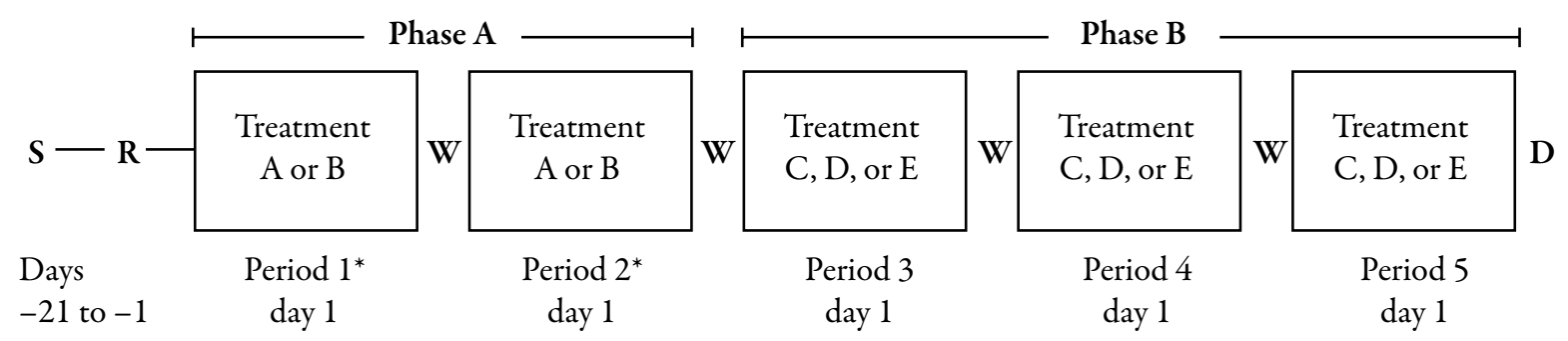

b)

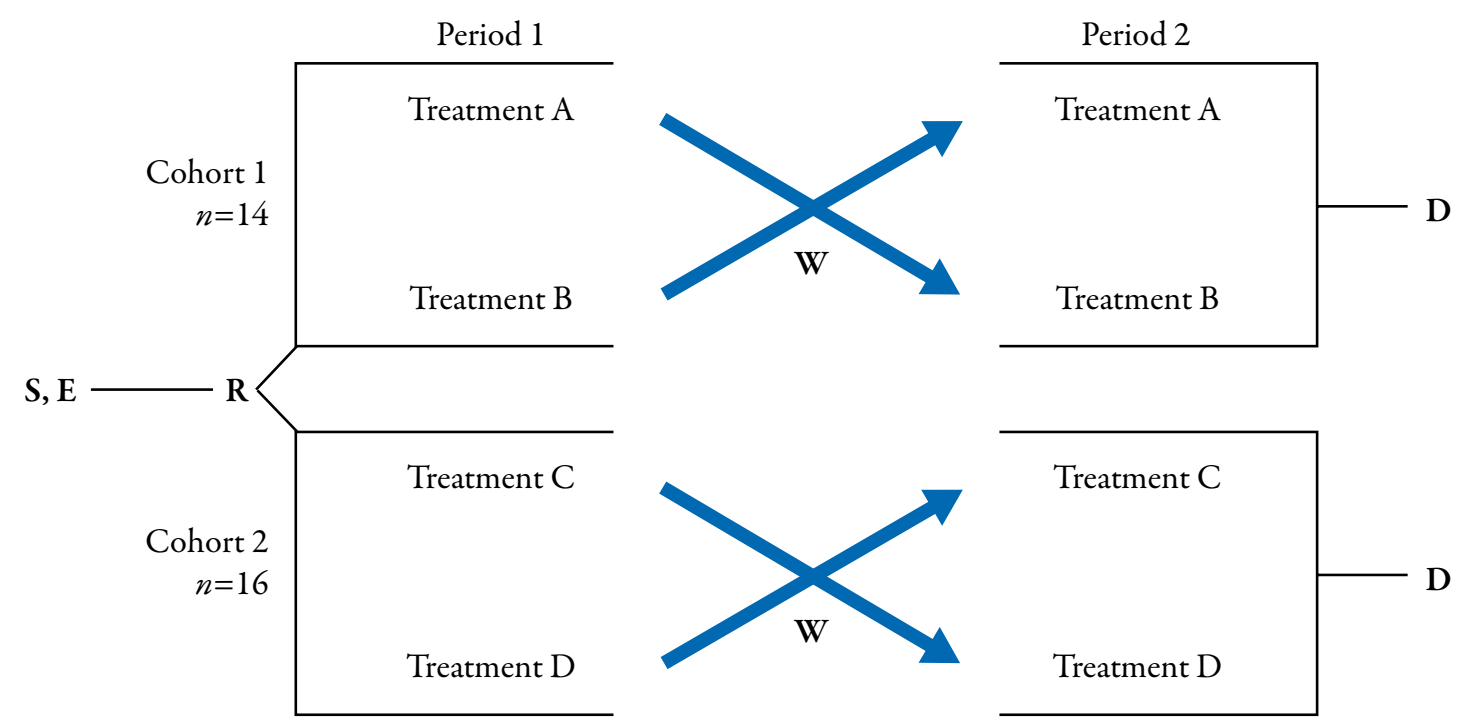

Day -28

$\begin{array}{lll}-1 & 1 & 2\end{array}$

8

Day $11 \quad 12$

8

11

Fig. 1. Design of studies to evaluate potential drug interactions of dapagliflozin with simvastatin, valsartan, warfarin, or digoxin. (a) Simvastatin or valsartan. Treatment A: $40 \mathrm{mg}$ simvastatin (single dose); treatment B: $20 \mathrm{mg}$ dapagliflozin (single dose) + $40 \mathrm{mg}$ simvastatin (single dose); treatment C: $20 \mathrm{mg}$ dapagliflozin (single dose); treatment D: $320 \mathrm{mg}$ valsartan (single dose); and treatment E: $20 \mathrm{mg}$ dapagliflozin (single dose) + $320 \mathrm{mg}$ valsartan (single dose). ${ }^{*}$ Pharmacokinetic collection for dapagliflozin and valsartan: 72 hours; for simvastatin/simvastatin acid: 48 hours. $D=$ study discharge; $\mathrm{R}=$ randomization; $\mathrm{S}=$ screening; $\mathrm{W}=\geq 4$-day washout. (b) Warfarin or digoxin. Treatment A: dapagliflozin $20 \mathrm{mg}$ tablet loading dose on day 1 followed by dapagliflozin $10 \mathrm{mg}$ tablet daily on days 2-8 and warfarin $25 \mathrm{mg}$ tablet on day 2 only; treatment B: warfarin $25 \mathrm{mg}$ tablet on day 2 only; treatment C: dapagliflozin $20 \mathrm{mg}$ tablet loading dose on day 1 followed by dapagliflozin $10 \mathrm{mg}$ tablet once daily on days $2-8$ and digoxin $0.25 \mathrm{mg}$ tablet on day 2 only; and treatment $\mathrm{D}$ : digoxin $0.25 \mathrm{mg}$ tablet on day 2 only. $\mathrm{D}=$ study discharge; $\mathrm{E}=$ enrollment; $\mathrm{R}=$ randomization; $\mathrm{S}=$ screening; $\mathrm{W}=$ washout 9 days relative to dosing for warfarin or digoxin.

with warfarin or digoxin to maintain sufficient exposure to dapagliflozin to allow adequate evaluation of a possible interaction between the drugs. The subjects in cohort 2 were randomized to receive either an oral loading dose of
$20 \mathrm{mg}$ dapagliflozin on day 1 followed by oral administration of $10 \mathrm{mg}$ dapagliflozin once daily for 7 days (days 2-8), and co-administration of a single oral dose of $0.25 \mathrm{mg}$ digoxin on day 2 , or a single oral dose of $0.25 \mathrm{mg}$ digoxin on day 2 . 
In both cohorts, subjects underwent a 9-day washout period that separated the single dose of either warfarin or digoxin. Fasting requirements and study drug intake with water were the same as for study 1 . Serial blood samples were collected in $\mathrm{K}_{2}$ EDTA tubes for up to 216 hours after dosing for pharmacokinetic assessment of R-warfarin, S-warfarin, and digoxin. Plasma was isolated by centrifugation and stored at $-20^{\circ} \mathrm{C}$ for dapagliflozin, R- and S-warfarin, and digoxin until analyzed.

\section{Drug Analyses}

Plasma concentrations of dapagliflozin; simvastatin; the active metabolite of simvastatin, simvastatin acid; valsartan; R-warfarin; S-warfarin; and digoxin were assayed by validated liquid chromatography-tandem mass spectrometry methods in analytical runs using appropriate calibration curves and quality control samples that met pre-established acceptance criteria within the known period of analyte stability. The assays were conducted in compliance with applicable standard operating procedures in place at the time of analysis. For study 1, all analyses were conducted by Cedra Corporation (Austin, TX, USA), and the respective between-run and within-run variability (lower limit of quantitation [LLOQ]; upper limit of quantitation [ULOQ]) of the assay were: for dapagliflozin $\leq 4.4 \%$ and $\leq 7.2 \%(1-200 \mathrm{ng} / \mathrm{mL})$; for simvastatin $\leq 2.5 \%$ and $\leq 5.8 \%(0.1-60 \mathrm{ng} / \mathrm{mL})$; for simvastatin acid $\leq 3.5 \%$ and $\leq 8.5 \%$ $(0.05-20 \mathrm{ng} / \mathrm{mL})$; and for valsartan $\leq 3.6 \%$ and $\leq 5.1 \%(10-8000 \mathrm{ng} / \mathrm{mL})$. For study 2 the dapagliflozin analysis was conducted at Cedra, and the warfarin and digoxin assays were conducted at PPD (Richmond, VA, USA). The between-run and within-run variability (LLOQ - ULOQ) of the assays were: for dapagliflozin $\leq 6.3 \%$ and $\leq 3.9 \%(1-200 \mathrm{ng} / \mathrm{mL})$; for R-warfarin $\leq 3.4 \%$ and $\leq 4.7 \%(5-1000 \mathrm{ng} / \mathrm{mL})$; for S-warfarin $\leq 3.0 \%$ and $\leq 5.0 \%(5-1000 \mathrm{ng} / \mathrm{mL})$; and for digoxin $\leq 8.4 \%$ and $\leq 14.2 \%(0.1-50 \mathrm{ng} / \mathrm{mL})$.

\section{Pharmacokinetic and Pharmacodynamic Evaluation}

Pharmacokinetic parameters were derived from plasma concentration versus time data and comprised maximum observed plasma concentration $\left(C_{\max }\right)$, time to maximum observed plasma concentration $\left(t_{\max }\right), t_{1 / 2,}$ and area under the plasma concentration versus time curve from time 0 to the last quantifiable concentration $\left(\mathrm{AUC}_{0-\tau}\right)$. The pharmacokinetic parameters were calculated using Kinetica ${ }^{\mathrm{TM}}$ version 4.4.1 in eToolbox EP version 2.6.1 (Thermo Electron Corporation, Philadelphia, PA, USA).

\section{Sample Collection}

Blood samples for the determination of dapagliflozin, simvastatin, simvastatin acid, and valsartan were taken at $0,0.5,1,1.5,2,3,4,6$, $8,12,16,24,36,48$, and 72 hours after dosing. For the determination of S- and R-warfarin and digoxin, blood samples were collected immediately prior to dosing and at $0.5,1,1.5$, $2,6,12,24,48,72,96,120,144,168,192$, and 216 hours after dosing on day 2.

At each collection time point, $4.0 \mathrm{~mL}$ blood samples were collected. Separate samples were collected into labeled $\mathrm{K}_{2}$ EDTA tubes for dapagliflozin, valsartan, R-warfarin, S-warfarin, and digoxin analysis. For the analysis of both simvastatin and simvastatin acid, samples were collected into labeled lithium-heparin tubes. Immediately after collection, each blood sample was gently inverted 8-10 times for complete mixing with the anticoagulant and placed on wet ice. Within 30 minutes of collection, blood 
samples were centrifuged at $5^{\circ} \mathrm{C}$ for 10 minutes at $1500 \times \mathrm{g}$. Within 15 minutes of centrifugation, the plasma was transferred into screw-capped polypropylene tubes and stored at or below $-20^{\circ} \mathrm{C}$ for dapagliflozin, R- and S-warfarin, and digoxin analyses and at or below $-70^{\circ} \mathrm{C}$ for valsartan, simvastatin, and simvastatin acid.

The pharmacodynamics of warfarin were derived by determining the plasma prothrombin time for treatments with warfarin and converting these to INR [31]. $\mathrm{AUC}_{(\mathrm{INR})}$ and maximum observed peak INR value $\left(\mathrm{INR}_{\max }\right)$ were calculated based on derived INR versus time data. The plasma prothrombin time measurements were assayed with a validated method by St. Luke's Hospital (Kansas City, MO, USA).

\section{Statistical Methods for Pharmacokinetic and Pharmacodynamic Analyses}

The sample sizes for the studies were not based on specific statistical power considerations. However, for study 1, data from 22 subjects would provide at least $90 \%$ confidence that the estimated ratios of geometric means for simvastatin $C_{\max }$ and $\mathrm{AUC}_{0-\tau}$, with or without dapagliflozin, would be within $17 \%$ and $24 \%$ of the true population ratios. For cohort 1 of study 2 , including 12 subjects provides at least $90 \%$ confidence that the estimated ratios of geometric means for S-warfarin $C_{\max }$ and $\mathrm{AUC}_{0-\tau}$, with or without dapagliflozin, would be within $10 \%$ and $11 \%$ of the true population ratios. For cohort 2 of study 2, including 14 subjects provides at least $90 \%$ confidence that the estimated ratios of geometric means for digoxin $C_{\max }$ and $\mathrm{AUC}_{0-\tau}$, with or without dapagliflozin, would be within $12 \%$ and $5 \%$ of the true population ratios.

To assess the effects of co-administration, point estimates and $90 \%$ CIs of the ratios of the adjusted geometric means for $C_{\max }$ and $\mathrm{AUC}_{0-\tau}$ of dapagliflozin given in combination with the cardiovascular drug under investigation and as monotherapy were calculated. The adjusted geometric means were obtained from general linear mixed models after accounting for all other effects in the model. Although not predefined, a 90\% CI with the usual bounds 0.80-1.25 was considered indicative of no effect.

To assess the effect of dapagliflozin on the pharmacodynamics of warfarin, point estimates and $90 \%$ CIs were calculated for warfarin alone and in the presence of dapagliflozin, and ratios of geometric means for $\mathrm{AUC}_{(\mathrm{INR})}$ and $\mathrm{INR}_{\max }$ were calculated based on appropriate general linear model analyses.

\section{Safety Profile}

Subjects were monitored throughout the study for all adverse events, with information recorded on intensity and relationship to study drug in the opinion of the investigator. Any serious adverse event was also recorded. Vital signs (body temperature, respiratory rate) and seated blood pressure and heart rate, measured after the subject had been seated quietly for $\geq 5$ minutes, were evaluated at the screening visit and prior to dosing on days 1, 2, and 3 of each period for study 1 , and on days $1,2,5$, and 8 for periods 1 and 2 for study 2 . Vital signs were also recorded on day -1 of periods $2,3,4$, and 5 for study 1 , as well as at study discharge for studies 1 and 2 . In addition, blood pressure was measured at the time of pharmacokinetic sample collection.

\section{RESULTS}

\section{Subject Demographics}

In study 1, 23 subjects were male and one was female; 63\% were African American, 29\% were white, and $8 \%$ were of other ethnicity. 
The mean age was 32 years (range, 21-43 years) and the mean BMI was 26.8 (range, 19.6-31.6). Twenty-three subjects completed study 1 . One subject discontinued the study because of agitation; this subject subsequently went on to have a serious adverse event.

In study 2, all 30 subjects were male. The predominant ethnic group was white (63\%), with African American (30\%) and other ethnic groups (7\%) accounting for the remainder. The mean age was 28 years (range, $19-45$ years) and the mean BMI was 25 (range, 18.7-31.5). One subject did not complete study 2 for personal reasons.

\section{Pharmacokinetics}

\section{Dapagliflozin}

The mean plasma concentration versus time profiles for dapagliflozin, with and without simvastatin or valsartan, were comparable (Fig. 2). No clinically meaningful changes in the pharmacokinetic parameters of dapagliflozin were observed when it was administered with either simvastatin or valsartan (Table 1).

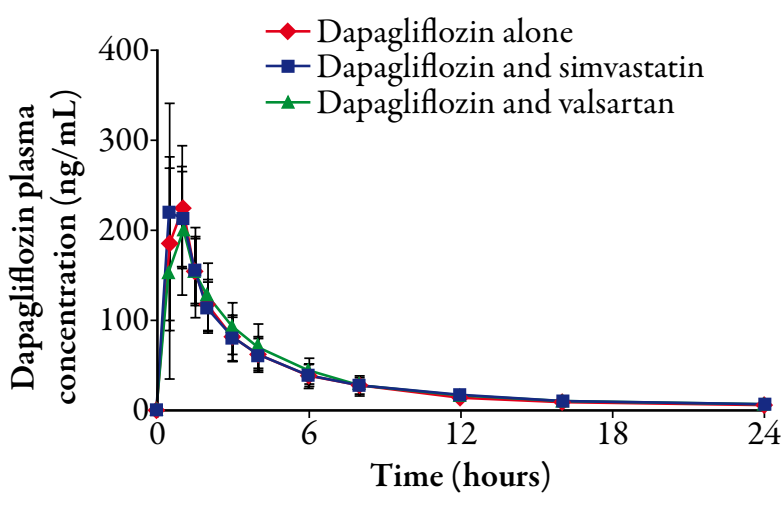

Fig. 2. Mean plasma concentration versus time profiles for dapagliflozin with and without co-administration of simvastatin or valsartan.

The $90 \%$ CIs for dapagliflozin $C_{\max }$ and $\mathrm{AUC}_{0-\tau}$ were within the usual no-effect interval of $0.8-1.25$. No changes that are likely to be clinically meaningful in the $t_{1 / 2}$ and $t_{\max }$ of dapagliflozin were observed when co-administered with either simvastatin or valsartan.

\section{Simvastatin, Simvastatin Acid, and Valsartan}

Mean plasma concentration versus time profiles for simvastatin, simvastatin acid, and valsartan are shown in Fig. 3.

Table 1. Pharmacokinetic parameters for dapagliflozin with and without simvastatin or valsartan.

\begin{tabular}{|c|c|c|c|c|c|}
\hline \multirow[b]{3}{*}{ Pharmacokinetic parameter } & \multicolumn{3}{|c|}{ Treatment } & \multicolumn{2}{|c|}{$\begin{array}{l}\text { Ratio of adjusted geometric } \\
\text { means point estimate }(90 \% \mathrm{CI})\end{array}$} \\
\hline & $\begin{array}{l}\text { Dapagliflozin } \\
(n=23)\end{array}$ & $\begin{array}{l}\text { Dapagliflozin } \\
\text { and simvastatin } \\
(n=24)\end{array}$ & $\begin{array}{l}\text { Dapagliflozin } \\
\text { and valsartan } \\
(n=24)\end{array}$ & & \\
\hline & A & B & $\mathrm{C}$ & $\mathrm{B}: \mathrm{A}$ & C:A \\
\hline$C_{\max }, \mathrm{ng} / \mathrm{mL}^{*}$ & 251 & 245 & 221 & $0.98(0.89-1.08)$ & $0.88(0.80-0.98)$ \\
\hline $\mathrm{AUC}_{0-\tau}, \mathrm{ng} \cdot \mathrm{h} / \mathrm{mL}^{*}$ & 928 & 906 & 947 & $0.98(0.95-1.01)$ & $1.02(1.00-1.05)$ \\
\hline$t_{\max }, \mathrm{h} \dagger$ & $0.55(0.50-2.03)$ & $1.00(0.50-2.00)$ & $0.99(0.48-2.00)$ & - & - \\
\hline$t_{1 / 2}, \mathrm{~h} \neq$ & $16.1(7.6)$ & $18.6(9.1)$ & $15.9(7.0)$ & - & - \\
\hline
\end{tabular}

${ }^{*}$ Adjusted geometric mean.

†Median (range).

$\neq$ Mean (SD).

$\mathrm{AUC}_{0-\tau}=$ area under the curve from time 0 to the last quantifiable concentration; $C_{\text {max }}=$ maximum observed plasma concentration; $t_{\max }=$ time to $C_{\max } ; t_{1 / 2}=$ half-life. 
a)

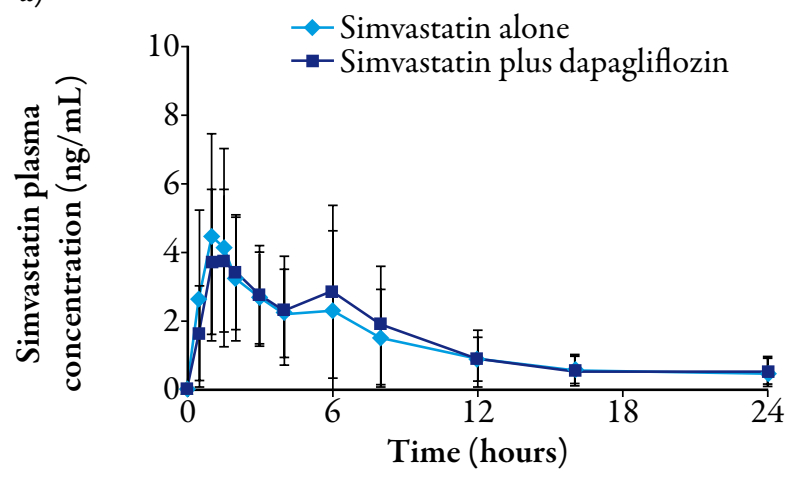

b)

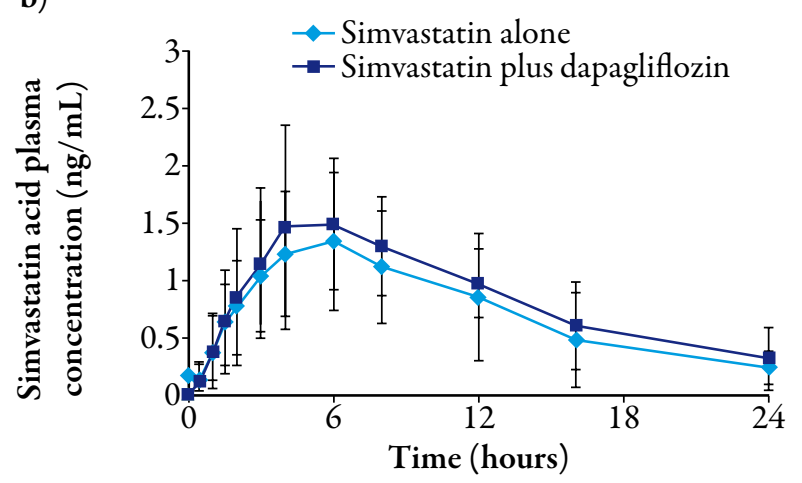

c)

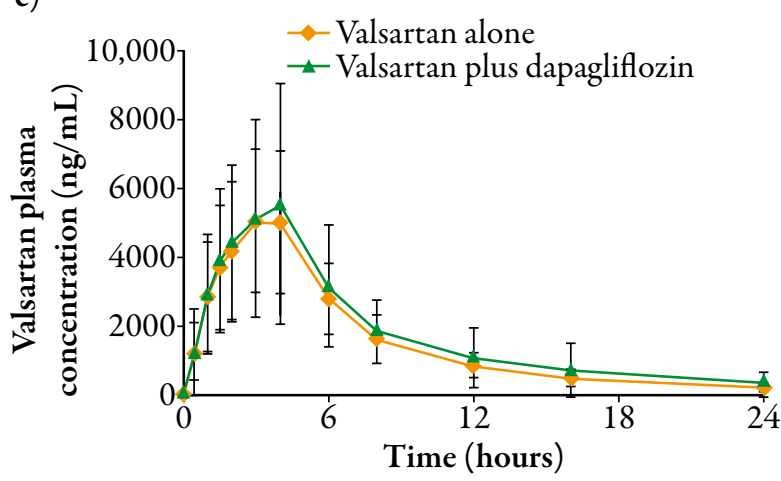

Fig. 3. Mean plasma concentration versus time profiles for (a) simvastatin, (b) its active metabolite, simvastatin acid, and (c) valsartan with and without co-administration of dapagliflozin.

There was no clinically meaningful change in simvastatin $C_{\max }$ in the presence of dapagliflozin. The $90 \%$ CI for the simvastatin $C_{\max }$ ratio fell within the usual no-effect range (Table 2). The $\mathrm{AUC}_{0-\tau}$ geometric mean for simvastatin increased by approximately $19 \%$ in the presence of dapagliflozin, and the upper limit of the $90 \%$ CI for the $\mathrm{AUC}_{0-\tau}$ ratio was slightly outside the usual 1.25 no-effect range (1.40).

Simvastatin acid $C_{\max }$ was also unaffected by co-administration with dapagliflozin, with the $90 \%$ CI for the $C_{\max }$ ratio falling within the usual no-effect range. The simvastatin acid $\mathrm{AUC}_{0-\tau}$ geometric mean increased by approximately $30 \%$, and the upper limit for the $90 \% \mathrm{CI}$ of the $\mathrm{AUC}_{0-\tau}$ ratio was slightly outside the usual no-effect range (1.47).

The geometric mean for valsartan $C_{\max }$ and $\mathrm{AUC}_{0-\tau}$ changed by no more than $6 \%$. When valsartan was co-administered with dapagliflozin, the $90 \%$ CI for the valsartan $C_{\max }$ ratio fell slightly outside the lower bound of the usual no-effect range (0.76), and the upper limit of the $90 \% \mathrm{CI}$ for the valsartan $\mathrm{AUC}_{0-\tau}$ ratio was slightly extended beyond the usual no-effect range (1.30).

\section{Warfarin and Digoxin}

Mean plasma concentration versus time profiles for R-warfarin, S-warfarin, and digoxin, with and without dapagliflozin, are shown in Fig. 4.

The geometric means for $C_{\max }$ and $\mathrm{AUC}_{0-\tau}$ for R-warfarin increased by no more than $8 \%$ when warfarin $25 \mathrm{mg}$ was administered in the presence of dapagliflozin $10 \mathrm{mg}$ relative to those observed following administration of warfarin $25 \mathrm{mg}$ alone (Table 3). The $90 \%$ CIs for the ratio of the geometric means for $C_{\max }$ and $\mathrm{AUC}_{0-\tau}$ for R-warfarin, with and without dapagliflozin, were all within the usual no-effect interval (Table 3). The geometric means for $C_{\max }$ and $\mathrm{AUC}_{0-\tau}$ for S-warfarin increased by $7 \%$ or less when warfarin $25 \mathrm{mg}$ was administered in the presence of dapagliflozin $10 \mathrm{mg}$ relative to those observed following administration of warfarin $25 \mathrm{mg}$ alone. The $90 \% \mathrm{CIs}$ for the ratio of population geometric means for $C_{\max }$ and $\mathrm{AUC}_{0-\tau}$ for S-warfarin, with and without dapagliflozin, were all within the usual no-effect interval. 
Table 2. Pharmacokinetic parameters for simvastatin, simvastatin acid, and valsartan, with and without dapagliflozin.

\begin{tabular}{|c|c|c|c|}
\hline \multirow[b]{2}{*}{ Pharmacokinetic parameter } & \multicolumn{2}{|c|}{ Treatment } & \multirow{2}{*}{$\begin{array}{l}\text { Ratio of adjusted geometric } \\
\text { means point estimate }(90 \% \mathrm{CI})\end{array}$} \\
\hline & Without dapagliflozin & With dapagliflozin & \\
\hline \multicolumn{4}{|l|}{ Simvastatin } \\
\hline$C_{\max }, \mathrm{ng} / \mathrm{mL}^{*}$ & 5.14 & 4.81 & $0.94(0.82-1.07)$ \\
\hline $\mathrm{AUC}_{0-\tau}, \mathrm{ng} \cdot \mathrm{h} / \mathrm{mL}^{*}$ & 25.21 & 29.90 & $1.19(1.01-1.40)$ \\
\hline$t_{\max }, \mathrm{h} \dagger$ & $1.50(0.48-8.00)$ & $1.99(1.00-6.03)$ & - \\
\hline$t_{1 / 2}, \mathrm{~h} \neq$ & $5.59(3.50)$ & $7.84(5.01)$ & - \\
\hline \multicolumn{4}{|l|}{ Simvastatin acid } \\
\hline$C_{\max }, \mathrm{ng} / \mathrm{mL}^{*}$ & 1.40 & 1.50 & $1.08(0.93-1.25)$ \\
\hline $\mathrm{AUC}_{0-\tau}, \mathrm{ng} \cdot \mathrm{h} / \mathrm{mL}^{*}$ & 16.40 & 21.27 & $1.30(1.15-1.47)$ \\
\hline$t_{\max }, \mathrm{h} \dagger$ & $6.00(2.00-12.00)$ & $6.00(3.00-411.97)$ & - \\
\hline$t_{1 / 2}, \mathrm{~h} \neq$ & $8.35(8.00)$ & $12.21(12.02)$ & - \\
\hline \multicolumn{4}{|l|}{ Valsartan } \\
\hline$C_{\max }, \mathrm{ng} / \mathrm{mL}^{*}$ & 5525 & 5184 & $0.94(0.76-1.16)$ \\
\hline $\mathrm{AUC}_{0-\tau}, \mathrm{ng} \cdot \mathrm{h} / \mathrm{mL}^{*}$ & 38,238 & 40,536 & $1.06(0.87-1.30)$ \\
\hline$t_{\max }, \mathrm{h} \dagger$ & $3.98(1.00-5.98)$ & $3.50(0.50-4.23)$ & - \\
\hline$t_{1 / 2}, \mathrm{~h} \neq$ & $16.79(18.60)$ & $13.47(9.88)$ & - \\
\hline
\end{tabular}

${ }^{*}$ Adjusted geometric mean.

tMedian (range).

$\neq$ Mean (SD).

$\mathrm{AUC}_{0-\tau}=$ area under the curve from time 0 to the last quantifiable concentration; $C_{\max }=$ maximum observed plasma concentration; $t_{\max }=$ time to $C_{\max } ; t_{1 / 2}=$ half-life.

The geometric means for $C_{\max }$ and $\mathrm{AUC}_{0-\tau}$ for digoxin differed by $1 \%$ or less when digoxin $0.25 \mathrm{mg}$ was administered in the presence of dapagliflozin relative to those observed following administration of digoxin $0.25 \mathrm{mg}$ alone. The $90 \%$ CIs for the ratios of geometric means, with and without dapagliflozin, were within the usual no-effect interval for digoxin $C_{\max }$ and $\mathrm{AUC}_{0-\tau}$.

\section{Pharmacodynamics of Warfarin}

The pharmacodynamics of warfarin were unaffected by the co-administration of dapagliflozin (Fig. 5). The geometric means for $\mathrm{INR}_{\max }$ and $\mathrm{AUC}_{(\mathrm{INR})}$ both increased by approximately $1 \%$, when warfarin was administered in the presence of dapagliflozin relative to those observed following administration of warfarin alone (Table 4). The $90 \% \mathrm{CI}$ for the ratio of $\mathrm{INR}_{\max }$ and $\mathrm{AUC}_{(\mathrm{INR})}$ between treatments fell within the usual no-effect range.

\section{Safety Profile}

Each drug and drug combination was well tolerated by the healthy subjects in these two studies. No serious adverse events, deaths, clinically relevant changes in vital signs or 12-lead electrocardiograms, or changes in physical examination findings were related to any of the study treatments. One subject experienced a serious adverse event that was not attributed to study treatment (psychiatric hospitalization) 24 days after being withdrawn 
a)

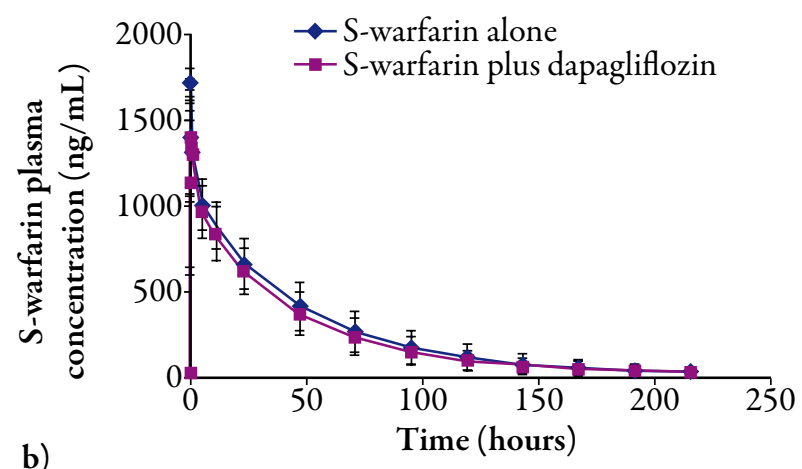

b)

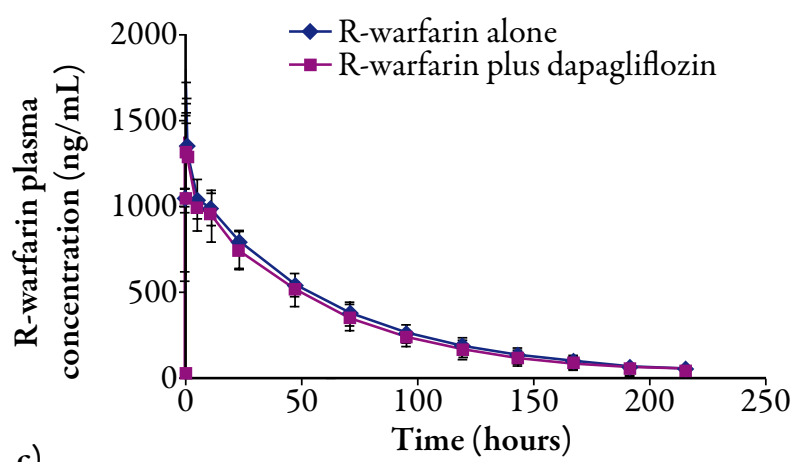

c)

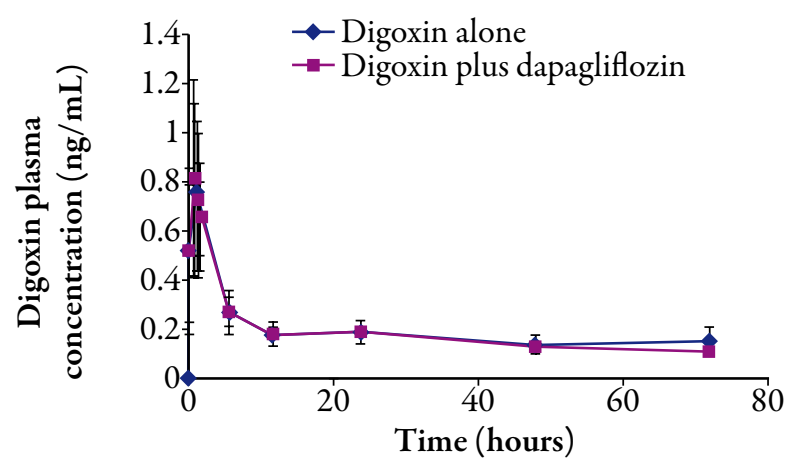

Fig. 4. Mean plasma concentration versus time profiles for (a) S-warfarin, (b) R-warfarin, and (c) digoxin, with and without co-administration of dapagliflozin.

from study 1 . No new clinically relevant safety signals emerged when drugs were given in combination in either study. A total of 34 adverse events were experienced by subjects in study 1 ; of these, 29 were mild and the remainder moderate. In study 2 , five subjects receiving a combination therapy experienced an adverse event. All of these events were mild.

Marked abnormalities in hematology, clinical chemistry, and urinalysis parameters were reported in 14 subjects in each study. The majority of the marked abnormalities were related to urine glucose, which is consistent with the pharmacologic effects of dapagliflozin. Other laboratory marked abnormalities were predominantly mild and transient, and the majority were resolved by the end of each of the studies.

\section{DISCUSSION}

Patients with T2DM, and in particular those requiring insulin, have an elevated risk for cardiovascular disease compared with the general population. This is due to an increase in the frequency of cardiovascular risk factors, such as dyslipidemia, hypertension, and abnormalities with blood clotting. It is estimated that approximately $70 \%-97 \%$ of patients with T2DM have diabetic dyslipidemia [32], 20\%-60\% have hypertension [33], and approximately $18 \%$ have atrial fibrillation [34]. The risk for suffering a stroke is approximately doubled for patients with T2DM over the general population [35]. Thus, patients diagnosed with T2DM often require the co-administration of several medications to manage not only glycemia, but also any associated cardiovascular risk factors. Pharmacokinetic or pharmacodynamic interactions between these co-administered medications may lead to changes in drug exposure or safety concerns.

Dapagliflozin is under development for T2DM and improves glycemic parameters in treatmentnaive patients [14], as well as in those requiring oral antihyperglycemic drugs $[13,14,36]$ or insulin [16]. As many of these patients, in particular those requiring insulin, would be expected to require cardiovascular drugs, it was necessary to establish the potential for interaction between dapagliflozin and commonly prescribed cardiovascular medications. 
Table 3. Pharmacokinetic parameters for R- and S-warfarin and digoxin, with and without dapagliflozin.

\begin{tabular}{|c|c|c|c|}
\hline \multirow[b]{2}{*}{ Pharmacokinetic parameter } & \multicolumn{2}{|c|}{ Treatment } & \multirow{2}{*}{$\begin{array}{l}\text { Ratio of adjusted geometric } \\
\text { means point estimate }(90 \% \mathrm{CI}\end{array}$} \\
\hline & Without dapagliflozin & With dapagliflozin & \\
\hline \multicolumn{4}{|l|}{ R-warfarin } \\
\hline$C_{\max }, \mathrm{ng} / \mathrm{mL}^{*}$ & 1438 & 1520 & $1.06(1.00-1.15)$ \\
\hline $\mathrm{AUC}_{0-\tau}, \mathrm{ng} \cdot \mathrm{h} / \mathrm{mL}^{*}$ & 68,006 & 73,195 & $1.08(1.03-1.12)$ \\
\hline$t_{\max }, \mathrm{h} \dagger$ & $1.00(0.50-2.00)$ & $1.00(0.50-2.00)$ & - \\
\hline$t_{1 / 2}, \mathrm{~h} \neq$ & $50.8(11.7)$ & $50.3(6.1)$ & - \\
\hline \multicolumn{4}{|l|}{$S$-warfarin } \\
\hline$C_{\max }, \mathrm{ng} / \mathrm{mL}^{*}$ & 1530 & 1575 & $1.03(0.99-1.12)$ \\
\hline $\mathrm{AUC}_{0-\tau}, \mathrm{ng} \cdot \mathrm{h} / \mathrm{mL}^{*}$ & 50,326 & 54,001 & $1.07(1.01-1.14)$ \\
\hline$t_{\max }, \mathrm{h} \dagger$ & $1.00(0.50-2.00)$ & $1.00(0.50-2.00)$ & - \\
\hline$t_{1 / 2}, \mathrm{~h} \neq$ & $49.3(11.8)$ & $44.9(6.3) \S$ & - \\
\hline \multicolumn{4}{|l|}{ Digoxin } \\
\hline$C_{\max }, \mathrm{ng} / \mathrm{mL}^{*}$ & 0.846 & 0.838 & $0.99(0.84-1.16)$ \\
\hline $\mathrm{AUC}_{0-\tau}, \mathrm{ng} \cdot \mathrm{h} / \mathrm{mL}^{*}$ & 9.479 & 9.496 & $1.00(0.86-1.17)$ \\
\hline$t_{\max }, \mathrm{h} \dagger$ & $1.00(0.50-2.00)$ & $1.00(0.50-2.02)$ & - \\
\hline
\end{tabular}

*Adjusted geometric mean.

†Median (range).

$\neq$ Mean $(\mathrm{SD})$.

$\S n=13$.

$\mathrm{AUC}_{0-\tau}=$ area under the curve from time 0 to the last quantifiable concentration; $C_{\text {max }}=$ maximum observed plasma concentration; $t_{\max }=$ time to $C_{\max } ; t_{1 / 2}=$ half-life.

This study evaluated the interaction of dapagliflozin with four commonly prescribed drugs, simvastatin, valsartan, warfarin, and digoxin.

The pharmacokinetic profile of dapagliflozin, as determined in study 1 , was consistent with profiles seen in previous monotherapy studies

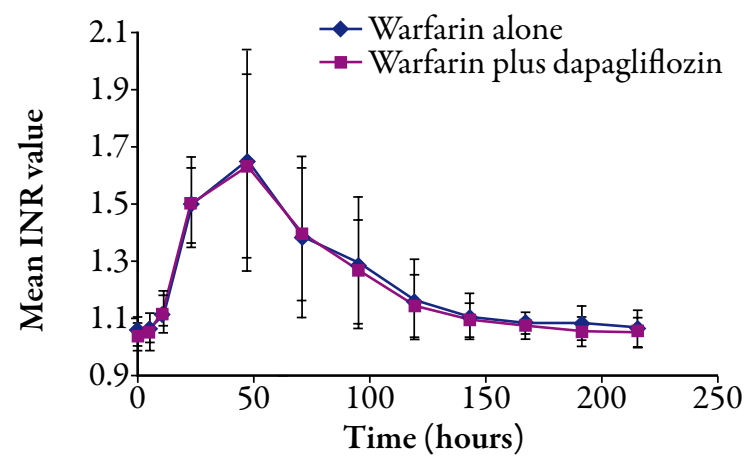

Fig. 5. Mean INR values versus time. INR=international normalized ratio. performed in healthy subjects [22], and the co-administration of either simvastatin or valsartan had no impact on the $C_{\max }$ of dapagliflozin or on the overall exposure to dapagliflozin, as assessed by $\mathrm{AUC}_{0-\tau}$. Similarly, the co-administration of dapagliflozin did not alter the $C_{\max }$ of simvastatin. The $90 \%$ CI was within the usual US FDA-defined no-effect range of $0.80-1.25$ [37], and the geometric mean ratio of simvastatin $\mathrm{AUC}_{0-\tau}$ was within the usual no-effect range, while the upper limit value was not within the no-effect range (1.40). Furthermore, the co-administration of dapagliflozin with simvastatin did not alter the $C_{\max }$ of simvastatin acid, although the geometric mean ratio of $\mathrm{AUC}_{0-\tau}$ was increased by $30 \%$. Co-administration of dapagliflozin did not appear to alter the $C_{\max }$ or $\mathrm{AUC}_{0-\tau}$ of valsartan. 
Table 4. International normalized ratios with and without dapagliflozin.

\begin{tabular}{llll}
\hline & \multicolumn{2}{c}{ Treatment } & Ratio of adjusted geometric \\
\cline { 2 - 3 } $\begin{array}{l}\text { Pharmacokinetic parameter } \\
\text { (geometric mean) }\end{array}$ & Without dapagliflozin & With dapagliflozin & means point estimate (90\% CI) \\
\hline $\mathrm{INR}_{\max }(\mathrm{CV} \%)$ & $1.65(21)$ & $1.64(18)$ & $1.004(0.967-1.043)$ \\
$\mathrm{AUC}_{(\mathrm{INR})}(\mathrm{CV} \%)$ & $268(10)$ & $265(8)$ & $1.007(0.989-1.025)$ \\
\hline $\mathrm{AUC}$ & &
\end{tabular}

$\mathrm{AUC}=$ area under the curve; $\mathrm{CV}=$ coefficient of variance.

While the 90\% CI values were not completely contained within the usual 0.80-1.25 no-effect interval, these changes were less than two-fold and would not warrant a dose adjustment of either drug based on FDA guidance. The findings of study 2 confirmed the expectation that the steady-state administration of dapagliflozin would be unlikely to have any substantial effect on the pharmacokinetics or pharmacodynamics of warfarin and the pharmacokinetics of digoxin.

Limitations of these studies include the predominantly-male (study 1) and all-male (study 2) populations studied, healthy subjects, small population, and single doses. These results are preliminary and larger clinical trials of longer duration in patients with T2DM would be required to support the evidence presented here. However, the results are considered applicable to patients with T2DM of either sex since marked sex differences are not observed for any of the drugs studied; the drug-drug interaction assessments are within-subject evaluations within the linear pharmacokinetic range of each drug; and none show time-dependent pharmacokinetics.

Dapagliflozin was well tolerated by the healthy subjects studied here when administered alone or in combination with simvastatin, valsartan, warfarin, or digoxin. Any adverse events were either mild or moderate in intensity. Clinical chemistry was closely monitored, and any marked abnormalities were generally mild in nature and had resolved by the conclusion of the studies. No changes in vital signs were observed throughout the course of these studies when dapagliflozin was co-administered with simvastatin, valsartan, warfarin, or digoxin.

Once-daily oral dapagliflozin has been shown to improve glycemic control without any increase in daily insulin requirements and also led to weight loss in patients with T2DM poorly controlled with insulin $[16,38]$. Patients such as these, with an elevated risk for cardiovascular disease, may benefit from insulin-independent approaches to controlling hyperglycemia, such as SGLT2 inhibition.

\section{CONCLUSION}

The absence of any pharmacokinetic interaction of dapagliflozin with simvastatin, valsartan, warfarin, or digoxin indicates that dapagliflozin may be safely co-administered with each of these cardiovascular drugs (and presumably other drugs with similar metabolism pathways). The data from the studies reported here, taken in combination with those from previous studies in which there was no pharmacokinetic interaction of dapagliflozin with drugs that had a variety of different metabolism pathways [39], may indicate a flexibility of use for dapagliflozin in a variety of clinical situations.

\section{ACKNOWLEDGMENTS}

This study was sponsored by Bristol-Myers Squibb and AstraZeneca. Medical writing assistance in 
the preparation of this manuscript was provided by Karen Pemberton, PhD, of PAREXEL and was funded by Bristol-Myers Squibb and AstraZeneca. Dr Kasichayanula is the guarantor for this article, and takes responsibility for the integrity of the manuscript as a whole.

The authors were shareholders and/or employees of Bristol-Myers Squibb at the time the study was conducted.

Open Access. This article is distributed under the terms of the Creative Commons Attribution Noncommercial License which permits any noncommercial use, distribution, and reproduction in any medium, provided the original author(s) and source are credited.

\section{REFERENCES}

1. International Diabetes Federation. The global burden. Available at: http://www.diabetesatlas.org/ book/export/html/36. Accessed October 12, 2010.

2. World Diabetes Foundation. Diabetes facts. Available at: http://www.worlddiabetesfoundation. org/composite-35.htm. Accessed September 1, 2010.

3. World Health Organization. Global strategy on diet, physical activity and health. Available at: http:// www.who.int/dietphysicalactivity/publications/ facts/obesity/en/. Accessed April 21, 2010.

4. Misra A, Ganda OP. Migration and its impact on adiposity and type 2 diabetes. Nutrition. 2007;23:696-708.

5. Bays HE, Chapman RH, Grandy S. The relationship of body mass index to diabetes mellitus, hypertension and dyslipidaemia: comparison of data from two national surveys. Int J Clin Pract. 2007;61:737-747.

6. Johansen OE, Birkeland KI. Preventing macrovascular disease in patients with type 2 diabetes mellitus. Am J Cardiovasc Drugs. 2003;3:283-297.

7. Nathan DM, Buse JB, Davidson MB, et al. Medical management of hyperglycemia in type 2 diabetes: a consensus algorithm for the initiation and adjustment of therapy: a consensus statement of the American Diabetes Association and the European Association for the Study of Diabetes. Diabetes Care. 2009;32:193-203.
8. Marsenic O. Glucose control by the kidney: an emerging target in diabetes. Am J Kidney Dis. 2009;53:875-883.

9. Meng W, Ellsworth BA, Nirschl AA, et al. Discovery of dapagliflozin: a potent, selective renal sodiumdependent glucose cotransporter 2 (SGLT2) inhibitor for the treatment of type 2 diabetes. J Med Chem. 2008;51:1145-1149.

10. Han S, Hagan DL, Taylor JR, et al. Dapagliflozin, a selective SGLT2 inhibitor, improves glucose homeostasis in normal and diabetic rats. Diabetes. 2008;57:1723-1729.

11. Komoroski B, Vachharajani N, Boulton D, et al. Dapagliflozin, a novel SGLT2 inhibitor, induces dose-dependent glucosuria in healthy subjects. Clin Pharmacol Ther. 2009;85:520-526.

12. Komoroski B, Vachharajani N, Feng Y, Li L, Kornhauser D, Pfister M. Dapagliflozin, a novel, selective SGLT2 inhibitor, improved glycemic control over 2 weeks in patients with type 2 diabetes mellitus. Clin Pharmacol Ther. 2009;85:513-519.

13. Bailey CJ, Gross JL, Pieters A, Bastien A, List JF. Effect of dapagliflozin in patients with type 2 diabetes who have inadequate glycaemic control with metformin: a randomised, double-blind, placebo-controlled trial. Lancet. 2010;375:2223-2233.

14. Ferrannini E, Ramos SJ, Salsali A, Tang W, List JF. Dapagliflozin monotherapy in type 2 diabetic patients with inadequate glycemic control by diet and exercise: a randomized, double-blind, placebo-controlled, phase III trial. Diabetes Care. 2010;33:2217-2224.

15. List JF, Woo V, Morales E, Tang W, Fiedorek FT. Sodium-glucose cotransport inhibition with dapagliflozin in type 2 diabetes. Diabetes Care. 2009;32:650-657.

16. Wilding JPH, Norwood $\mathrm{P}, \mathrm{T}^{\prime}$ joen $\mathrm{C}$, Bastien A, List JF, Fiedorek FT. A study of dapagliflozin in patients with type 2 diabetes receiving high doses of insulin plus insulin sensitizers. Applicability of a novel insulin-independent treatment. Diabetes Care. 2009;32:1656-1662.

17. Wilding JPH, Woo V, Soler NG, Pahor A, Sugg J, Parikh S. Sustained effectiveness of dapagliflozin over 48 weeks in patients with type 2 diabetes poorly controlled with insulin [abstract]. Diabetes. 2010;59(Suppl. 1). Abstract 0021-LB.

18. Stratton IM, Adler AI, Neil HA, et al. Association of glycaemia with macrovascular and microvascular complications of type 2 diabetes (UKPDS 35): prospective observational study. BMJ. 2000;321:405-412. 
19. Laakso M. Hyperglycemia and cardiovascular disease in type 2 diabetes. Diabetes. 1999;48:937-942.

20. Bayturan O, Tuzcu EM, Uno K, et al. Comparison of rates of progression of coronary atherosclerosis in patients with diabetes mellitus versus those with the metabolic syndrome. Am J Cardiol. 2010;105:1735-1739.

21. Nichols GA, Koro CE, Gullion CM, Ephross SA, Brown JB. The incidence of congestive heart failure associated with antidiabetic therapies. Diabetes Metab Res Rev. 2005;21:51-57.

22. Obermeier $M$, Yao $M$, Khanna $A$, et al. In vitro characterization and pharmacokinetics of dapagliflozin (BMS-512148), a potent sodiumglucose cotransporter type II inhibitor, in animals and humans. Drug Metab Dispos. 2010;38:405-414.

23. Nakashima A, Kawashita H, Masuda N, et al. Identification of cytochrome P450 forms involved in the 4-hydroxylation of valsartan, a potent and specific angiotensin II receptor antagonist, in human liver microsomes. Xenobiotica. 2005;35:589-602.

24. Prueksaritanont T, Gorham LM, Ma B, et al. In vitro metabolism of simvastatin in humans [SBT] identification of metabolizing enzymes and effect of the drug on hepatic P450s. Drug Metab Dispos. 1997;25:1191-1199.

25. Garnett WR. Interactions with hydroxymethylglutaryl-coenzyme A reductase inhibitors. Am J Health Syst Pharm. 1995;52:1639-1645.

26. Kaminsky LS, Zhang ZY. Human P450 metabolism of warfarin. Pharmacol Ther. 1997;73:67-74.

27. Hinderling PH, Hartmann D. Pharmacokinetics of digoxin and main metabolites/derivatives in healthy humans. Ther Drug Monit. 1991;13:381-401.

28. Gatmaitan ZC, Arias IM. Structure and function of P-glycoprotein in normal liver and small intestine. Adv Pharmacol. 1993;24:77-97.

29. Simonson SG, Martin PD, Mitchell PD, Lasseter K, Gibson G, Schneck DW. Effect of rosuvastatin on warfarin pharmacodynamics and pharmacokinetics. J Clin Pharmacol. 2005;45:927-934.
30. O'Reilly RA. Studies on the optical enantiomorphs of warfarin in man. Clin Pharmacol Ther. 1974;16:348-354.

31. Stern R, Karlis V, Kinney L, Glickman R. Using the international normalized ratio to standardize prothrombin time. J Am Dent Assoc. 1997;128:1121-1122.

32. Gold JA. Managing diabetic dyslipidemia: testing is step one. WMJ. 2004;103:51, 54.

33. American Diabetes Association. Treatment of hypertension in adults with diabetes. Diabetes Care. 2002;25:199-201.

34. Dublin S, Glazer NL, Smith NL, et al. Diabetes mellitus, glycemic control, and risk of atrial fibrillation. J Gen Intern Med. 2010;25:853-858.

35. Bruno A, Liebeskind D, Hao Q, Raychev R. Diabetes mellitus, acute hyperglycemia, and ischemic stroke. Curr Treat Options Neurol. 2010;12:492-503.

36. Strojek K, Hruba V, Elze M, Langkilde A, Parikh S. Efficacy and safety of dapagliflozin in patients with type 2 diabetes mellitus and inadequate glycaemic control on glimepiride monotherapy [abstract]. Diabetologia. 2010;53(Suppl. 1):S347.

37. US Food and Drug Administration. Guidance for industry drug interaction studies-study design, data analysis, and implications for dosing and labeling. Available at: http://www.fda.gov/Drugs/ GuidanceComplianceRegulatoryInformation/ Guidances/ucm064982.htm. Accessed August 14, 2011.

38. Soler NG, Wilding JPH, Woo V, et al. Dapagliflozin lowered rate of insulin uptitration/study discontinuation from lack of glycaemic control in 48-week study of type 2 diabetes patients poorly controlled on insulin therapy [abstract]. Diabetologia. 2010;53(Suppl. 1):S348-S349. Abstract 872.

39. Kasichayanula S, Liu X, Shyu WC, et al. Lack of pharmacokinetic interaction between dapagliflozin, a novel SGLT2 inhibitor, and metformin, pioglitazone, glimepiride, or sitagliptin in healthy subjects. Diabetes Obes Metab. 2011;13:47-54. 